\title{
European survey on the opinion and use of micronutrition in age-related macular degeneration: 10 years on from the Age-Related Eye Disease Study
}

This article was published in the following Dove Press journal:

Clinical Ophthalmology

10 October 2014

Number of times this article has been viewed

\author{
Tariq Aslam' \\ Cécile Delcourt ${ }^{2}$ \\ Frank Holz ${ }^{3}$ \\ Alfredo García-Layana ${ }^{4}$ \\ Anita Leys ${ }^{5}$ \\ Rufino M Silva ${ }^{6}$ \\ Eric Souied ${ }^{7}$ \\ 'Manchester Royal Eye Hospital, \\ Manchester, UK; ${ }^{2}$ University \\ of Bordeaux, Bordeaux, France; \\ ${ }^{3}$ University of Bonn, Bonn, Germany; \\ ${ }^{4}$ Clínica Universidad de Navarra, \\ Pamplona, Spain; ${ }^{5}$ University Hospitals, \\ Leuven, Belgium; 'University \\ of Coimbra, Coimbra, Portugal; \\ 'Université Paris Est Créteil, Créteil, \\ France
}

Purpose: To evaluate ophthalmologists' opinion of, and use of, micronutritional dietary supplements 10 years after publication of the first Age-Related Eye Disease Study (AREDS) study.

Methods: Participation was solicited from 4,000 European ophthalmologists. Responding physicians were screened, and those treating at least 40 patients with age-related macular degeneration (AMD) per month and prescribing nutrition supplements at least 4 times per month were admitted and completed a 40-item questionnaire.

Results: The surveyed sample included 112 general ophthalmologists and 104 retinal specialists. Most nutritional supplements (46\%) were initiated when early/intermediate AMD was confirmed, although $18 \%$ were initiated on confirmation of neovascular AMD. Clinical studies were well known: 90\% were aware of AREDS, with 88\% aware of AREDS1 and 36\% aware of the, as-yet-unpublished, AREDS2 studies. Respondents considered lutein, zeaxanthin, zinc, omega-3, and vitamins to be the most important components of nutritional supplements, with the results of AREDS2 already having been taken into consideration by many. Ophthalmologists anticipate more scientific studies as well as improved product quality but identify cost as a barrier to wider uptake.

Conclusion: Micronutrition is now part of the routine management of AMD for many ophthalmologists. Ophthalmologists choosing to use nutritional supplements are well-informed regarding current scientific studies.

Keywords: age-related macular degeneration, micronutrition, nutritional supplementation

\section{Introduction}

Age-related macular degeneration (AMD) is the main cause of blindness in the developed world, with the number of patients affected being counted in the millions and likely to increase as populations age. ${ }^{1,2}$ Risk factors for the development of the disease are well-established. ${ }^{3}$ Although antivascular endothelial growth factor (anti-VEGF) treatments have improved the outlook for many patients, a burden of morbidity remains.

Considerable advances in the understanding of the pathogenesis of AMD have been made in recent years, and the interactions between aging and genetic and environmental factors are being clarified. ${ }^{4}$ One of the environmental factors believed to be involved is oxidative stress in the retina. ${ }^{5,6}$ This forms the biological basis for a number of studies that have investigated the use of dietary supplementation (particularly with vitamins and antioxidants) ${ }^{7-13}$ the best known and most comprehensive of which are Age-Related Eye Disease Study (AREDS) and AREDS2. ${ }^{8,14,15}$ AREDS revealed a significant effect of zinc and antioxidants on the development of advanced AMD in patients with early signs of the disease and recommended their use in at-risk
Correspondence:Tariq Aslam Research Centre, 4th Floor, Manchester Royal Eye Hospital, Oxford Road, Manchester MI3 9WH, UK

Tel +44 I6I 2765619

Email tariq.aslam@manchester.ac.uk 
patients. AREDS 2 examined further the effects of carotenoids and omega-3 long-chain fatty acids in patients at risk for AMD.

The objective of this current study, performed before the issue of AREDS 2 results, was to assess the opinion and use of micronutrient supplements by European ophthalmologists who are somehow involved in the prescription of nutritional supplements or in providing advice for nutritional supplements to their AMD patients. In particular, the study seeks to profile the ophthalmologists and their clinical practice, to identify their attitudes to dietary supplements and the extent of their knowledge of scientific developments, and to characterize their expectations of this field in the future. Such information would be of great use in planning resources and identifying areas in which evidence-based approaches are not being implemented. Although the case for properly conducted clinical trials is unarguable, practical issues in the day-to-day management of AMD should not be ignored.

\section{Methods}

A total of 4,000 office or hospital-based ophthalmologists from Belgium, France, Germany, Italy, Portugal, Spain, and the UK were initially contacted by email. Ophthalmologists were eligible for inclusion if they were treating a minimum of 40 AMD patients per month, writing or giving at least four prescriptions or advice per month for nutritional supplements, and were not involved in remunerated work for pharmaceutical companies or other health care-related companies (other than as investigators in clinical trials). Respondents were stratified to provide an approximately equal balance of self-declared generalists and retinal specialist ophthalmologists. The first 30 eligible ophthalmologists in each country who responded were included.

Those ophthalmologists who were included completed an Internet-based questionnaire devised by the Committee of European Experts on Micronutrition of the Eye in their local language.

\section{Results}

\section{Survey population demographics and practice characteristics}

Of the 4,000 ophthalmologists contacted, 249 agreed to participate and 216 (around 30 per country) were eligible and entered into the survey (112 general ophthalmologists and 104 retinal specialists). Thirty-three ophthalmologists were excluded because they did not give at least 15 prescriptions or dietary advice each month. Demographic characteristics of the survey population are summarized in Table 1 .

The age distribution of the participants was unremarkable, other than a small preponderance of retinal specialists in the 51-60 years old category.

The proportion of ophthalmologists having public, private, or both public and private practices was similar between general ophthalmologists and retinal specialists.

A large proportion (78\%) of the ophthalmologists performed factor anti-VEGF injections on their AMD patients

Table I Practice characterization

\begin{tabular}{|c|c|c|}
\hline & $\begin{array}{l}\text { General ophthalmologists, } \% \\
(\mathbf{n}=\text { I I 2) }\end{array}$ & $\begin{array}{l}\text { Retinal specialists, } \% \\
(n=104)\end{array}$ \\
\hline \multicolumn{3}{|l|}{ Age distribution, years } \\
\hline$<30$ & 2 & I \\
\hline $30-40$ & 42 & 44 \\
\hline $4 I-50$ & 27 & 37.5 \\
\hline $51-60$ & 22 & 17.5 \\
\hline $61-70$ & 7 & - \\
\hline \multicolumn{3}{|l|}{ Practice type } \\
\hline Public & 27 & 29 \\
\hline Private & 12.5 & II.5 \\
\hline Both & 60.5 & 59.5 \\
\hline Mean total number of patients seen per month & 365 & 356 \\
\hline Mean number age-related macular degeneration patients seen per month & 71 & 119 \\
\hline Mean number nutritional supplement prescriptions written per month & 40 & 34 \\
\hline Mean number of oral advice on nutritional supplements given per month & 40 & 64 \\
\hline $\begin{array}{l}\text { Ophthalmologists undertaking anti-vascular endothelial growth } \\
\text { factor injections }\end{array}$ & 66 & 90 \\
\hline $\begin{array}{l}\text { Average number of anti-vascular endothelial growth factor injections } \\
\text { performed per month per ophthalmologist (for the } 78 \% \text { who personally } \\
\text { perform anti-vascular endothelial growth factor injections) }\end{array}$ & 22 & 45.5 \\
\hline
\end{tabular}


(Table 1). Unsurprisingly, this proportion was greater in the retinal specialists $(90 \%)$ compared with general ophthalmologists $(66 \%)$. The number of anti-VEGF injections administered per month was 35 for the group as a whole and 22 and 46 for general ophthalmologists and retinal specialists, respectively. There were notable international variations in the administration of anti-VEGF injections: whereas German ophthalmologists were the least likely to perform injections $(53.1 \%)$, the number of injections performed by those ophthalmologists who did perform them was the highest (71.5 per month). French ophthalmologists were the most likely to perform anti-VEGF injections, but the number of injections per month was near the group average. The proportion of ophthalmologists from Italy, Spain, and Portugal performing anti-VEGF injections was near the group average $(70 \%, 83.3 \%$, and $75.5 \%$, respectively), but the number of injections per month was considerably lower than for the group as a whole $(13.5,23.1$, and 19.3, respectively).

\section{AMD patient population}

\section{Severity distribution}

Retinal specialists saw more patients with neovascular disease than did general ophthalmologists. Overall, 39\% of patients had late-stage disease (geographic atrophy or neovascular): $41 \%$ among general ophthalmologists and 37.5\% among retinal specialists.

\section{Origin of patients}

The mean number of newly diagnosed patients each month by the ophthalmologists was 23 . This number was somewhat lower for general ophthalmologists (18.5) and somewhat higher for retinal specialists (28). Ophthalmologists from Italy, Belgium, and Portugal diagnosed fewer patients per month than the group average $(12.7,14.2$, and 10.4, respectively), whereas French and, particularly, UK ophthalmologists diagnosed more new patients (30.5 and 37.9 per month, respectively).

The pattern of AMD patient recruitment was different between general ophthalmologists and retinal specialists: general ophthalmologists diagnosed AMD among a higher proportion of their own patients than did retinal specialists (56\% versus $37 \%$, respectively). However, retinal specialists diagnosed more patients referred from a general ophthalmologist ( $28 \%$ versus $12 \%$, respectively) or from an optician or optometrist ( $28 \%$ versus $11.5 \%$, respectively).

There were notable differences between countries in the source of new patients: In the UK, $44.1 \%$ of new patients were referrals from opticians or optometrists, whereas in most countries, they were less than $5 \%$.

The average number of patients seen each month was 361 , with only small differences between general ophthalmologists and retinal specialists (Table 1), although there were significant differences between countries, with French, German, and Belgian ophthalmologists seeing significantly more patients per month $(513,462$, and 420 patients per month, respectively) and with Italian and Portuguese ophthalmologists seeing significantly fewer patients (209 and 206 patients per month, respectively) than the group average.

In a typical month, 89 nutritional supplements were prescribed or oral advice provided (37 written prescriptions, 52 oral advice; Table 1). There were notable differences between countries, with the greatest number of prescriptions written in a month observed in France and Spain (69.2 in both countries; $P<0.01$ versus the group average); the smallest number was observed in Belgium, Italy, and Portugal (14.5, 26 , and 23.6, respectively; all $P<0.01$ versus the group average). German ophthalmologists provided the greatest amount of oral advice, whereas Italian ophthalmologists provided the least ( 94.5 versus 18.9 , respectively).

The majority of patients underwent visual acuity $(99 \%$ early-intermediate stage, $98 \%$ geographic atrophy stage, $99 \%$ neovascular stage), funduscopy ( $95 \%$ early-intermediate stage, 94\% geographic atrophy stage, 95\% neovascular stage) and optical coherence tomography (70\% early-intermediate stage, $62 \%$ geographic atrophy stage, $91 \%$ neovascular stage) testing. Fluorescein angiography was frequently performed in neovascular-stage patients (71\% versus $35 \%$ and $27 \%$ in early-intermediate stage and geographic atrophy stage, respectively), whereas genetic testing was rarely performed (4\% in all AMD stages). There were a large number of differences between the tests administered by country, although consistent trends are not apparent.

The frequency of ophthalmology visits per year varied with disease severity and treatment. Early-intermediate-stage and geographic atrophy-stage patients visited their ophthalmologists an average of 2.6 and 2.5 times per year, respectively. The mean numbers of visits for neovascular-stage patients treated with anti-VEGF or not were 8.8 and 5.6 times per year, respectively. Similar findings were observed in the different countries.

More than three-quarters of patients $(78 \%)$ were prescribed anti-VEGF treatments $(81.8 \%$ retinal specialists and $73.6 \%$ general ophthalmologists). Reasons for not prescribing anti-VEGF included clinical symptoms too advanced (41\%), patient refusal $(20 \%)$, patient too old or in poor 
health $(16 \%)$, contraindication (15\%), cost $(7 \%)$, and other ( $1 \%$; patients preferred photodynamic therapy, referred to retinal specialist).

\section{Survey results}

\section{Ophthalmologist}

Overall, $65 \%$ of patients with AMD were currently receiving nutritional supplements (69\% general ophthalmologists, 60\% retinal specialists). This percentage was highest in Portugal (78.3\%) and Spain (72.2\%) and was lowest in Germany and the UK (both 52.6\%).

The interviewed ophthalmologist was the most common person to initiate the primary prescription or provide advice on nutritional supplements $(68 \% ; 71 \%$ general ophthalmologist, $63 \%$ retinal specialist). The same was true for all the countries, and in particular France (73.7\%) and Italy (74.7\%), where the index ophthalmologist was responsible for somewhat more prescription initiations than the group average. Another ophthalmologist was responsible for most of the remainder of the primary prescriptions $(20 \%)$. The ophthalmologist was also the most common health care professional to renew nutritional supplements $(61 \%)$. The findings were the same in all of the countries except the UK, where the general practitioner most frequently renewed nutritional supplements $(63.7 \%$ versus 19\% for ophthalmologists).

Nutritional supplementation was most frequently initiated when diagnosis of early or intermediate AMD was confirmed
( $46 \%$ of patients; $49 \%$ general ophthalmologist, $42 \%$ retinal specialist), followed by $18 \%$ (15\% general ophthalmologist, $21 \%$ retinal specialist) and $13 \%$ (12\% general ophthalmologist, $15 \%$ retinal specialist) of patients when neovascular AMD or geographic atrophy was confirmed, respectively. Only $3 \%$ of patients (2.8\% general ophthalmologist, $1.5 \%$ retinal specialist) were initiated on nutritional supplementation when anti-VEGF treatment was started.

The most important risk factors for initiating treatment with nutritional supplements were AMD stage, smoking, family history, and known genetic risk factors (Figure 1). Patient's age and nutrition were also essential factors.

Medical interest in nutritional supplements was important at all stages of the disease but was greatest in early/ intermediate disease, at 78\% (relatively high and very high medical interest) versus 58\% for neovascular AMD, and 59\% for geographic atrophy. Relatively similar opinions were observed in the different countries.

\section{Patient}

According to the ophthalmologist, more than half of their patients $(58 \% ; 59 \%$ general ophthalmologist, 56\% retinal specialist) who were taking nutritional supplements were not aware of them before receiving advice or their first prescription. Lack of patient awareness was greatest in Portugal (69.7\%) and Spain (67.5\%). Italian and UK patients more frequently raised an interest in nutritional supplements with the ophthalmologist (38.33\% and $35.0 \%$, respectively).

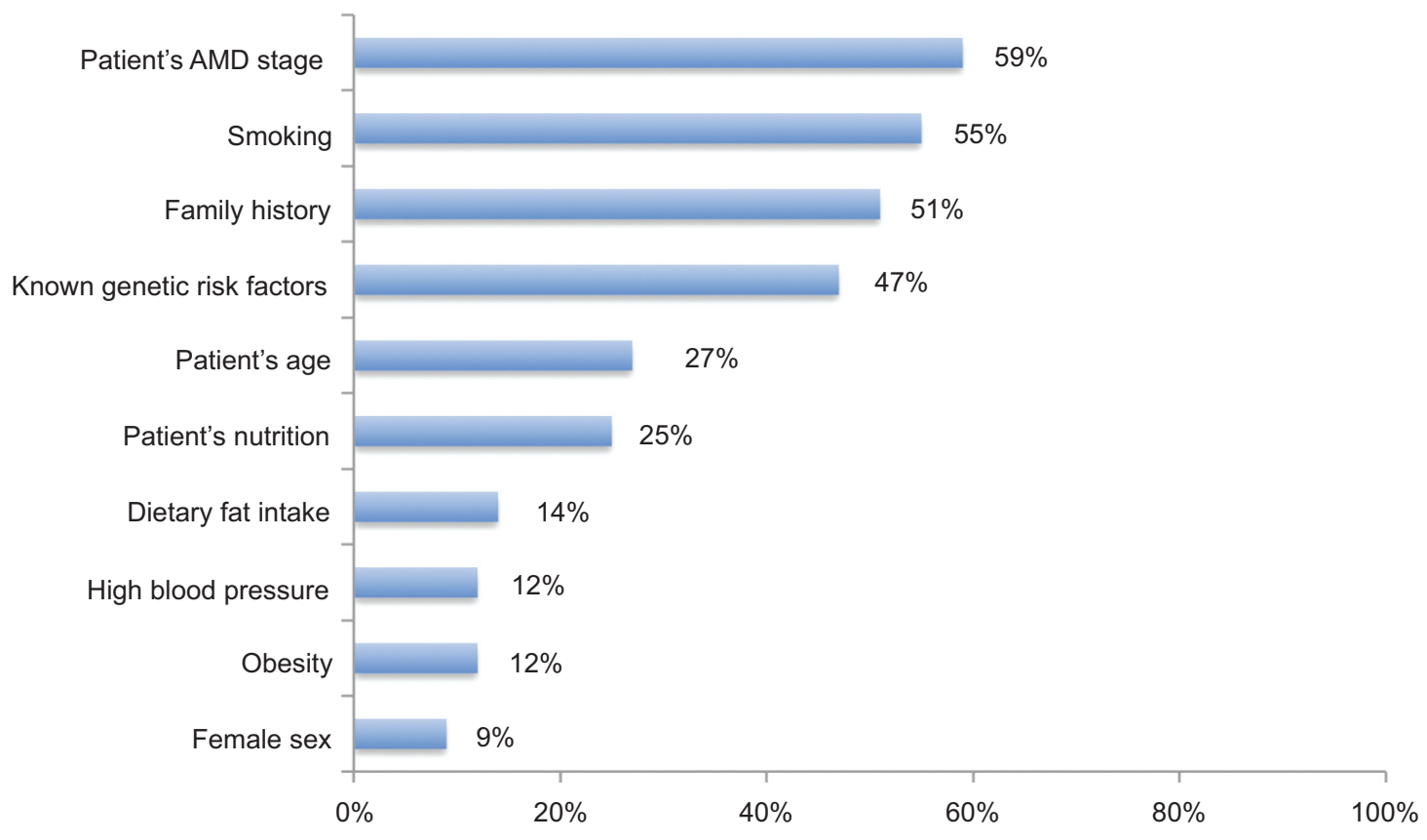

Figure I Very important risk factors for the initiation of nutritional supplementation. Abbreviation: AMD, age-related macular degeneration. 


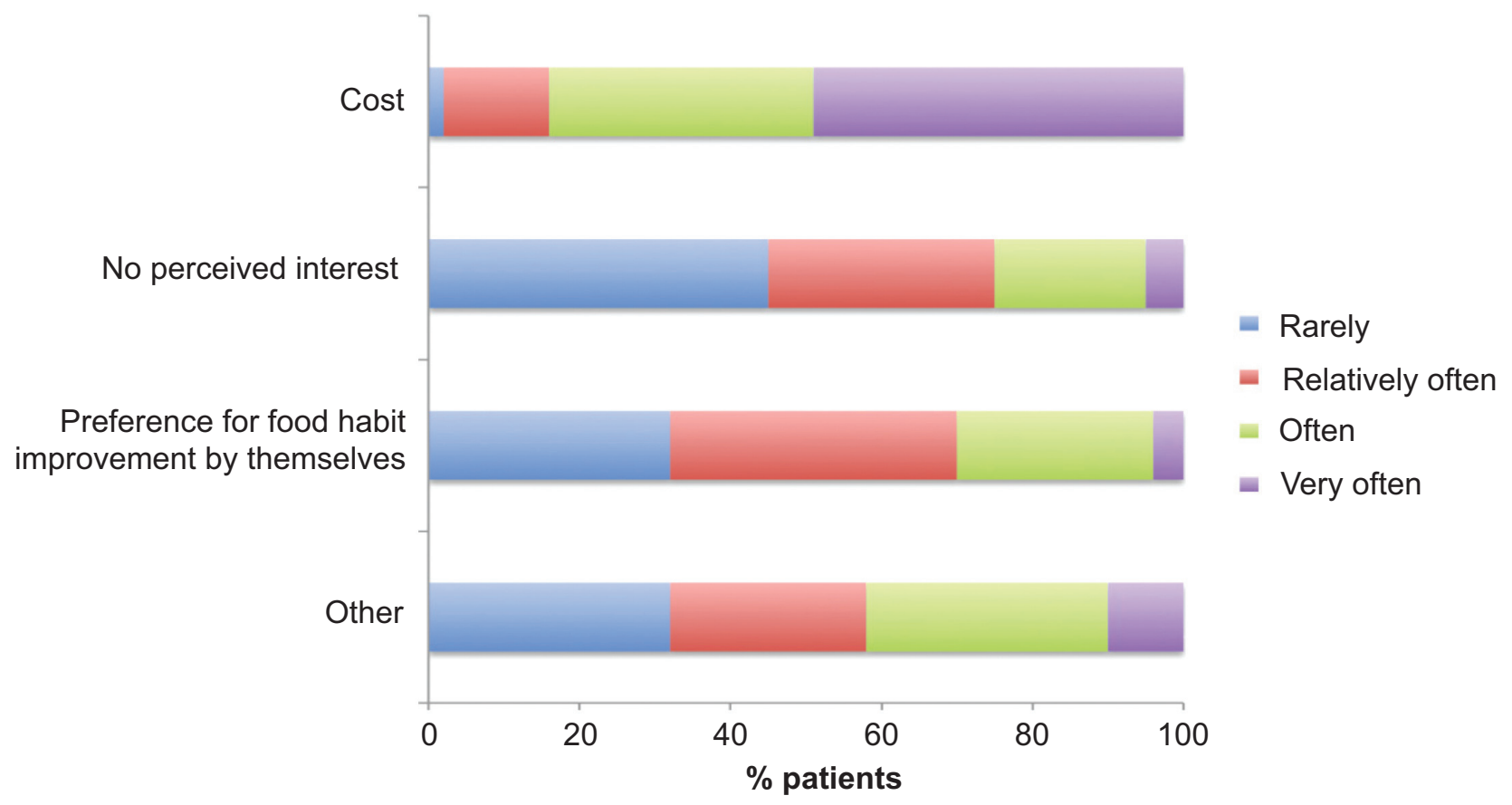

Figure 2 Reasons for patients refusing nutritional supplements.

Direct requests for nutritional supplements were highest in Germany (19.5\%).

Information concerning the beneficial effects of nutritional supplements was regularly given by $67 \%$ of ophthalmologists (75\% general ophthalmologist, 59\% retinal specialist); information was less regularly given in Spain $(40.0 \%)$ and the UK (53.3\%).

Patients rarely refused nutritional supplements (average, $14 \%$ for early/intermediate stage, $13 \%$ for geographic atrophy stage, $11 \%$ for neovascular stage), although where they did refuse, cost was the main reason (Figure 2).

Ophthalmologists suspected that $40 \%$ of patients had poor compliance ( $43 \%$ general ophthalmologist, $37 \%$ retinal specialist). Compliance improved with advanced disease (only $29 \%$ of patients with neovascular disease had poor compliance) and if patients were seen by retinal specialists (poor compliance reported by $46 \%$ versus $40 \%$ in early/ intermediate disease by general ophthalmologists and retinal specialists, respectively).

\section{Evidence and expectations}

A total of $83 \%$ of ophthalmologists were aware of any clinical studies related to the use of nutritional supplements in AMD ( $86 \%$ general ophthalmologist, $81 \%$ retinal specialist). Most ophthalmologists had heard of the AREDS (90\% [88.5\% general ophthalmologist, $91.7 \%$ retinal specialist]; AREDS 1, $88 \%$ [ $87.5 \%$ general ophthalmologist, $88.1 \%$ retinal specialist]; AREDS 2, 36\% [39.6\% general ophthalmologist, $32.1 \%$ retinal specialist]). AREDS 1 and 2 were rated of high medical interest (14.9 and 15.5 on a scale of 1 to 20, with $1=$ no interest and $20=$ extremely high interest), followed by clinical studies in general (11.9) and preclinical in vitro/ animal studies (6.4 and 6.5).

Ophthalmologists were generally of the opinion that nutritional supplements were effective in slowing disease progression in early/intermediate-stage AMD (Table 2). Expectations in terms of stopping disease progression and reducing lesions or damage were lower, particularly in geographic and neovascular stages.

Table 2 General opinion regarding efficacy of nutritional supplements in age-related macular degeneration

\begin{tabular}{|c|c|c|c|}
\hline & Early/intermediate stages & Geographic atrophy stage & Neovascular atrophy stage \\
\hline To slow progression & 14/20 (14.5 versus I3.7) & $10.2 / 20(10.5$ versus 9.9$)$ & $8.7 / 20$ (8.8 versus 8.7$)$ \\
\hline To stop progression & $8.2 / 20(8.8$ versus 7.6$)$ & $6.0 / 20(6.5$ versus 5.5$)$ & $5.0 / 20(5.3$ versus 4.7$)$ \\
\hline To reduce lesions, damage & $8.5 / 20$ (8.7 versus 8.2$)$ & $6.5 / 20(6.5$ versus 6.5$)$ & $6.1 / 20(6.2$ versus 6.0$)$ \\
\hline
\end{tabular}

Notes: I= very bad opinion; $20=$ excellent/best possible opinion. Numbers in parentheses are general ophthalmologist versus retinal specialist scores. 
Only $21.8 \%$ of ophthalmologists $(15.2 \%$ general ophthalmologist, $28.8 \%$ retinal specialist) considered nutritional supplements to have no symptomatic benefit. Nutritional supplements were considered to have the most beneficial symptomatic effects on visual acuity $(62 \% ; 66.1 \%$ general ophthalmologist, $56.7 \%$ retinal specialist), contrast vision $(58 \% ; 65.2 \%$ general ophthalmologist, $51.0 \%$ retinal specialist), and glare $(31 \% ; 33 \%$ general ophthalmologist, $27.9 \%$ retinal specialist). Nutritional supplements were expected to have a positive effect on both eyes, particularly in early/intermediate-stage disease.

There was a wide spectrum of opinions regarding the most important components for nutritional supplements (Figure 3). The most important spontaneous answers were lutein $(77 \%$; 81\% general ophthalmologist, $72 \%$ retinal specialist), omega-3 (72\%; 79\% general ophthalmologist, $64 \%$ retinal specialist), and zeaxanthin $(68 \% ; 72 \%$ general ophthalmologist, $62 \%$ retinal specialist). Among the list of all the components, lutein, omega-3, zeaxanthin, zinc, and vitamin $\mathrm{E}$ were considered as very important by more than $50 \%$ of respondents. Resveratrol was considered to be a very important component by $26 \%$ of ophthalmologists (30\% general ophthalmologist, $21 \%$ retinal specialist). Although a pattern is difficult to discern, there were important national differences in the importance attached to various components of nutritional supplements. The most striking was that $90.6 \%$ of German ophthalmologists considered lutein to be an important component compared with the group average of $77 \%$. In the UK, vitamins and fatty acids (including docosahexaenoic acid and eicosapentaenoic acid) were generally rated lower than other components, whereas French ophthalmologists rated pigments (71.9\%) and vitamin $\mathrm{D}(34.4 \%)$ considerably higher than the group averages $(33 \%$ and $13 \%$, respectively). As well as interesting differences between individual countries and the group averages, there were also notable contrasts between individual countries: German and Belgian ophthalmologists considered neoxanthin and astaxanthin as among the least-important components (3.1\% and $6.3 \%$ among the German ophthalmologists, respectively, and $6.7 \%$ and $3.3 \%$, respectively, among the Belgian ophthalmologists compared with $56.7 \%$ and $43.3 \%$ of Italian ophthalmologists who considered them important). Belgian ophthalmologists were generally the most skeptical (identifying six of the 19 proposed substances as significantly $[P<0.05]$ less important than the group average and rating none as significantly more important than the group average). In contrast, Italian ophthalmologists identified eleven of the 19 substances as being significantly more important than group average and none as less important than the group average), among them resveratrol (56.7\% versus the overall average of $26 \%$; Table 3 ).

Cost, scientific support, and product characteristics were believed the most important components when considering a nutritional supplement (Figure 4). More scientific research

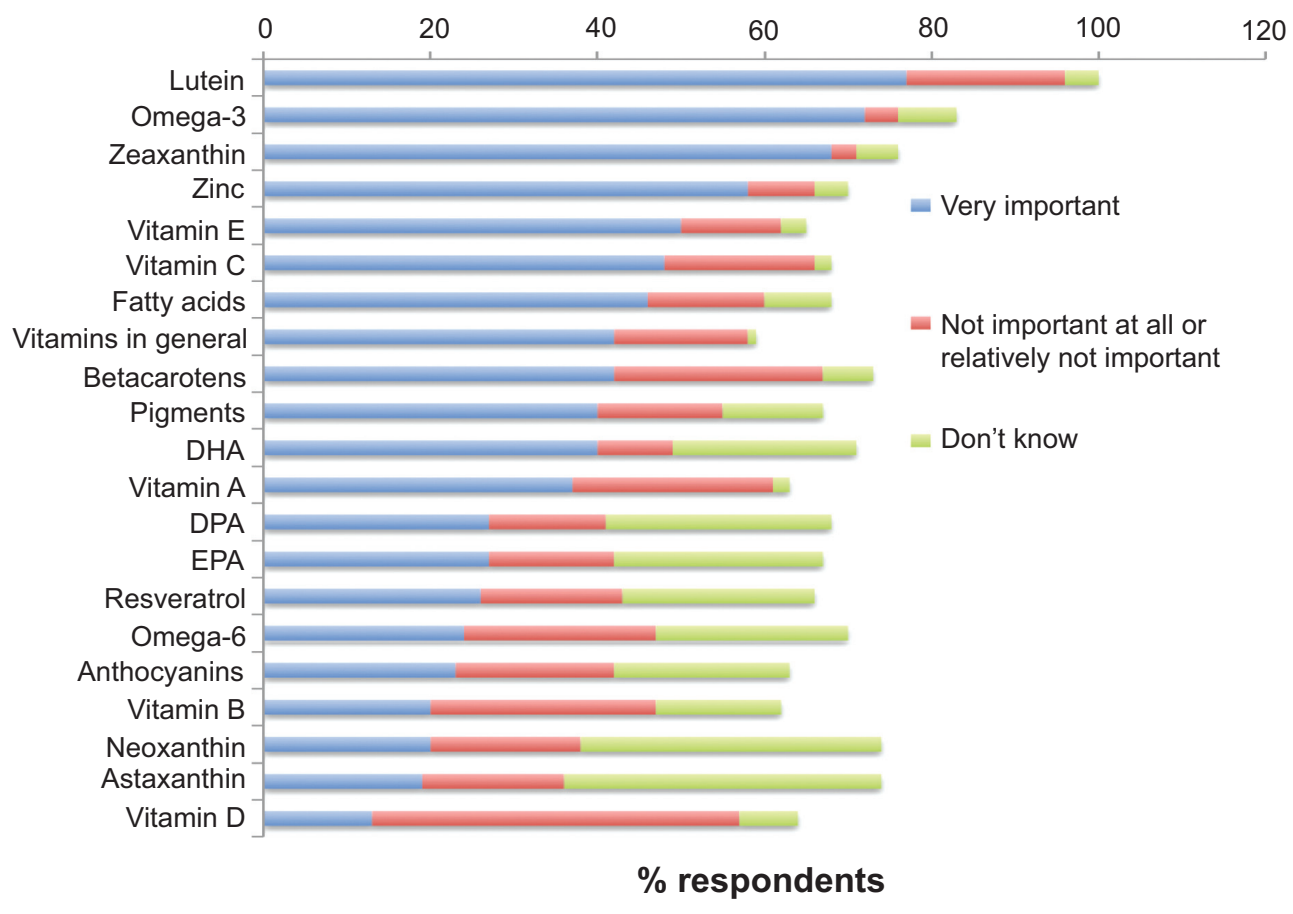

Figure 3 Important components in nutritional supplements.

Abbreviations: DHA, docosahexaenoic acid; DPA, docosapentaenoic acid: EPA, eicosapentaenoic acid. 
Table 3 National differences in important components of nutritional supplements

\begin{tabular}{|c|c|c|c|c|c|c|c|}
\hline Very important & $\begin{array}{l}\text { France } \\
(n=32)\end{array}$ & $\begin{array}{l}\text { Germany } \\
(n=32)\end{array}$ & $\begin{array}{l}\text { Italy } \\
(n=30)\end{array}$ & $\begin{array}{l}\text { Spain } \\
(n=30)\end{array}$ & $\begin{array}{l}\text { United Kingdom } \\
(n=30)\end{array}$ & $\begin{array}{l}\text { Belgium } \\
(\mathrm{n}=30)\end{array}$ & $\begin{array}{l}\text { Portugal } \\
(n=32)\end{array}$ \\
\hline Lutein & $75.0 \%$ & $90.6 \%(+)$ & $70.0 \%$ & $73.3 \%$ & $83.3 \%$ & $63.3 \%$ & $81.3 \%$ \\
\hline Omega-3 & $71.9 \%$ & $78.1 \%$ & $80.0 \%$ & $66.7 \%$ & $60.0 \%$ & $66.7 \%$ & $78.1 \%$ \\
\hline Zeaxanthin & $62.5 \%$ & $78.1 \%$ & $56.7 \%$ & $63.3 \%$ & $70.0 \%$ & $66.7 \%$ & $75.0 \%$ \\
\hline Zinc & $59.4 \%$ & $56.3 \%$ & $76.7 \%(+)$ & $46.7 \%$ & $60.0 \%$ & $60.0 \%$ & $46.9 \%$ \\
\hline Vitamin E & $43.8 \%$ & $53.1 \%$ & $83.3 \%(++)$ & $40.0 \%$ & $40.0 \%$ & $33.3 \%$ & $53.1 \%$ \\
\hline Vitamin C & $56.3 \%$ & $59.4 \%$ & $73.3 \%(++)$ & $26.7 \%(-)$ & $33.3 \%$ & $33.3 \%$ & $53.1 \%$ \\
\hline Fatty acids & $62.5 \%(+)$ & $50.0 \%$ & $60.0 \%$ & $33.3 \%$ & $20.0 \%(--)$ & $26.7 \%(-)$ & $65.6 \%(+)$ \\
\hline Vitamins (in general) & $46.9 \%$ & $56.3 \%$ & $53.3 \%$ & $43.3 \%$ & $30.0 \%$ & $30.0 \%$ & $31.3 \%$ \\
\hline Beta-carotene & $31.3 \%$ & $37.5 \%$ & $70.0 \%(++)$ & $36.7 \%$ & $46.7 \%$ & $30.0 \%$ & $43.8 \%$ \\
\hline Pigments & $71.9 \%(++)$ & $37.5 \%$ & $40.0 \%$ & $33.3 \%$ & $50.0 \%$ & $13.3 \%(--)$ & $34.4 \%$ \\
\hline Docosahexaenoic acid & $53.1 \%$ & $31.3 \%$ & $50.0 \%$ & $43.3 \%$ & $20.0 \%(-)$ & $40.0 \%$ & $43.8 \%$ \\
\hline Eicosapentaenoic acid & $37.5 \%$ & $28.1 \%$ & $43.3 \%(+)$ & $16.7 \%$ & $3.3 \%(--)$ & $33.3 \%$ & $28.1 \%$ \\
\hline Resveratrol & $25.0 \%$ & $15.6 \%$ & $56.7 \%(++)$ & $20.0 \%$ & $26.7 \%$ & $10.0 \%(-)$ & $28.1 \%$ \\
\hline Omega-6 & $15.6 \%$ & $25.0 \%$ & $40.0 \%(+)$ & $36.7 \%$ & $20.0 \%$ & $16.7 \%$ & $15.6 \%$ \\
\hline Anthocyanins (blueberries) & $25.0 \%$ & $18.8 \%$ & $53.5 \%(++)$ & $23.3 \%$ & $13.3 \%$ & $13.3 \%$ & $15.6 \%$ \\
\hline Vitamin B & $21.9 \%$ & $21.9 \%$ & $43.3 \%(++)$ & $16.7 \%$ & $6.7 \%(-)$ & $10.0 \%$ & $18.8 \%$ \\
\hline Neoxanthin & $15.6 \%$ & $3.1 \%(--)$ & $56.7 \%(++)$ & $26.7 \%$ & $13.3 \%$ & $6.7 \%(-)$ & $18.8 \%$ \\
\hline Astaxanthin & $21.9 \%$ & $6.3 \%(-)$ & $43.3 \%(++)$ & $26.7 \%$ & $16.7 \%$ & $3.3 \%(-)$ & $15.6 \%$ \\
\hline Vitamin D & $34.4 \% * *$ & $15.6 \%$ & $16.7 \%$ & $6.7 \%$ & $3.3 \%(-)$ & $3.3 \%(-)$ & $12.5 \%$ \\
\hline
\end{tabular}

Notes: "How important are each of the following components when choosing a food supplement product for age-related macular degeneration patients?" Values significantly greater or lower, respectively, than the group average are indicated as follows: $+/-=P<0.05,++/--=P<0.0$, Fisher-Snedecor test.

(29\%), improved efficacy (26\%), and lower cost (26\%) were thought the most important future expectations for the use of nutritional supplementation in AMD. The availability of new products was less frequently identified as an expectation (11\%), along with small pill size and lower dosing frequency. More communications campaigns and the development of new modes of administration were rated as expectations by $10 \%$ or fewer of the respondents.

\section{Discussion and conclusion}

This study examined the attitudes of ophthalmologists who prescribe at least some nutritional supplements to patients with AMD. The sample is not representative of ophthalmologists in general, as only those who followed at least 50 AMD patients per month and who prescribed supplements at least 15 times per month were included. In this sense, the sample could be considered biased, but the intention was to review

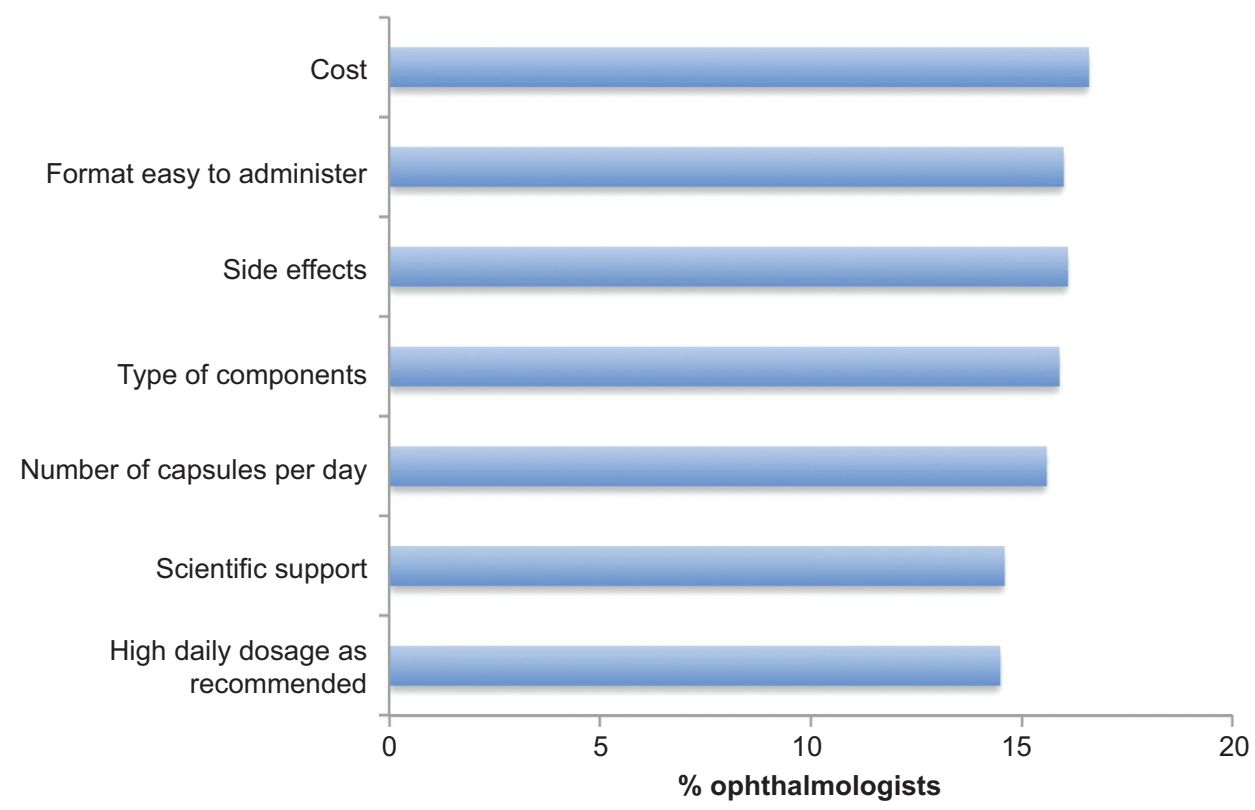

Figure 4 Important criteria in the decision-making process ( $I=$ not important at all; $20=$ extremely important). 
the attitudes and background of retina specialists and general ophthalmologists with some experience as nutritional supplement prescribers.

It would appear that even in the current nascent stage of clinical research, micronutrition has already become part of the day-to-day management of AMD for a considerable proportion of ophthalmologists; supplements were prescribed for $65 \%$ of the sample's patients overall, although there were considerable geographic differences ranging from more than three-quarters of the ophthalmologists Portugal prescribing supplements, to around half in the UK and Germany. Although ophthalmologists most frequently initiated the prescription in all countries, in the UK, the general practitioner was most likely to renew the prescription.

Nutritional supplementation was most commonly commenced in early and intermediate disease, a finding that matches the expectations of the clinicians that were higher for early-stage disease. Moreover, only small numbers of patients started nutritional supplementation after the commencement of anti-VEGF therapy. Poor compliance was seen as common, and in most cases, this was judged to be a result of cost and was worse in patients with early-stage disease. Refusal of nutritional supplementation was relatively rare (in the 10\%-15\% range, and lower in patients with more advanced disease). Ophthalmologists seem pragmatic in their expectations regarding the effect of nutritional supplements, with slowing of progression in the early stages of the disease being the strongest expectation, although more than half expected to see slowing of progression in the geographic atrophy stage. Arresting progression during the later stages of disease was much less expected. At all stages of the disease, a majority of respondents expected an effect in both eyes, although the proportion decreased with advancing disease and there was a corresponding increase in the proportion of respondents expecting improvement in the other eye alone.

The sampled ophthalmologists had a relatively sophisticated understanding of the scientific background to micronutritional supplementation. The great majority (90\% overall) were aware of AREDS, and more than one-third were aware of the as-yet-unpublished AREDS 2. Smaller numbers were aware of other studies. AREDS 1 and AREDS 2 were both considered of high medical interest, as were clinical studies in general. Clinical studies were considered the most interesting, with preclinical studies being rated of interest around half as frequently.

There was much less agreement on what constitutes the most important components of nutritional supplement.
Lutein, zeaxanthin, zinc, and vitamin E were mentioned by more than half the ophthalmologists. The heterogeneity of opinion is well illustrated by the lutein, which was considered very important by $77 \%$ of ophthalmologists (the highest proportion of any of the substance); however, lutein was also considered "not important at all or relatively not important" by $19 \%$ of ophthalmologists (only four other substances were considered unimportant by a higher proportion of responders). The results suggest that although the participants were aware of the results of the original AREDS study, ${ }^{7}$ they had anticipated the results of AREDS 2, whose methodology ${ }^{15}$ had been published but, at the time of the questionnaire, not its results, which showed no clear benefit for omega-3 supplementation. ${ }^{8}$

Although there are significant differences between countries in many of the parameters, the majority of the differences are attributable to different health care environments as well as medical custom and practice. In Germany, for example, the great majority of ophthalmologists have both public and private practice, and none have solely private practice. In the UK, there were no solely private ophthalmological practitioners, but all had at least some private practice. Practitioners in France, Germany, and Belgium see twice as many patients per month as do those in Italy and Portugal. The greater role of the general practitioner in the UK system is also evident, as the number of renewals for nutritional supplement prescriptions by general practitioner is almost three times greater in the UK than in any other country, and five times greater than all but Italy. Doubtless, these differences in custom and practice, as well as in reimbursement systems, are the drivers for many of the other differences observed between countries. However, although differences exist, some of which are significant, general trends are hard to identify. The particular choice by the respondents of substance that could or should be included in nutritional supplements for AMD suggests inhomogeneous knowledge and understanding of the scientific and clinical background. This may be understandable because of the large list of substances, and particularly as the results of AREDS 2 were not available when the questionnaire was administered.

The practitioners seem to have specific expectations for the development of the field. There was a clear preference for more scientific, and specifically clinical, data. These expectations will have been, at least in part, met in the meantime by the publication of AREDS 2, despite the ongoing controversy regarding its results. Expectations in terms of product quality and cost were also clear. Cost was identified as an issue limiting compliance, as nutritional supplements are not 
reimbursed, and moreover, patient awareness was relatively low, providing another compliance-limiting factor. These results indicate areas in which patient pathways to treatment, and education in particular, might lead to improvements in real-world treatment.

Overall, the ophthalmologists comprising this sample of nutritional supplement prescribers have a clear interest and well-defined expectations in developments in this field. They appear to have reasonable expectations of the benefits of nutritional supplements and have already adapted their practice to the emerging clinical results, and will presumably continue to do so in the light of AREDS 2. Although there are obvious differences between general ophthalmologists and retinal specialists and between ophthalmologists from different nations, these differences seem relatively small in the overall context.

\section{Acknowledgments}

The study was sponsored by Laboratoires Thea. Dr JF Stolz assisted in the preparation of the manuscript; this assistance was reimbursed by Laboratoires Thea.

\section{Disclosure}

TA has received travel awards and payment for advisory boards from Laboratoires Thea and Bausch and Lomb. CD acts as a consultant for Laboratoires Thea, Novartis, and Bausch and Lomb. FH has received honoraria, travel grants, or other disbursements from Acucela, Alcon, Genentech, Hoffmann-La Roche, Bayer, Novartis, Heidelberg Engineering, Optos, and Laboratoires Thea. AGL has received disbursements for consultancy from Alcon, Allergan, Bayer Novartis, and Laboratoires Thea. AL has received travel grants from Bausch + Lomb, Bayer, Laboratoires Thea, and Novartis and honoraria from Laboratoires Thea. RMS has received honoraria for participating in advisory boards for Alimera, Allergan, Bayer, Laboratoires Thea, and Novartis. ES has received travel grants or honoraria from Allergan, Bausch and Lomb, Bayer, Novartis, and Laboratoires Thea.

Clinical Ophthalmology

\section{Publish your work in this journal}

Clinical Ophthalmology is an international, peer-reviewed journal covering all subspecialties within ophthalmology. Key topics include: Optometry; Visual science; Pharmacology and drug therapy in eye diseases; Basic Sciences; Primary and Secondary eye care; Patient Safety and Quality of Care Improvements. This journal is indexed on

\section{References}

1. Bressler NM. Age-related macular degeneration is the leading cause of blindness. JAMA. 2004;291(15):1900-1901.

2. Friedman DS, O'Colmain BJ, Muñoz B, et al; Eye Diseases Prevalence Research Group. Prevalence of age-related macular degeneration in the United States. Arch Ophthalmol. 2004;122(4):564-572.

3. Chakravarthy U, Wong TY, Fletcher A, et al. Clinical risk factors for age-related macular degeneration: a systematic review and metaanalysis. BMC Ophthalmol. 2010;10:31.

4. Miller JW. Age-related macular degeneration revisited - piecing the puzzle: the LXIX Edward Jackson memorial lecture. Am J Ophthalmol. 2013;155(1):1-35.

5. Zarbin MA. Current concepts in the pathogenesis of age-related macular degeneration. Arch Ophthalmol. 2004;122(4):598-614.

6. Beatty S, Koh H, Phil M, Henson D, Boulton M. The role of oxidative stress in the pathogenesis of age-related macular degeneration. Surv Ophthalmol. 2000;45(2):115-134.

7. Querques G, Benlian P, Chanu B, et al. Nutritional AMD treatment phase I (NAT-1): feasibility of oral DHA supplementation in age-related macular degeneration. Eur J Ophthalmol. 2009;19(1):100-106.

8. Age-Related Eye Disease Study 2 Research Group. Lutein + zeaxanthin and omega-3 fatty acids for age-related macular degeneration: the Age-Related Eye Disease Study 2 (AREDS2) randomized clinical trial. JAMA. 2013;309(19):2005-2015.

9. Richer S. ARMD - pilot (case series) environmental intervention data. J Am Optom Assoc. 1999;70(1):24-36.

10. Zeimer M, Dietzel M, Hense HW, Heimes B, Austermann U, Pauleikhoff D. Profiles of macular pigment optical density and their changes following supplemental lutein and zeaxanthin: new results from the LUNA study. Invest Ophthalmol Vis Sci. 2012;53(8):4852-4859.

11. Ying GS, Huang J, Maguire MG, et al; Comparison of Age-related Macular Degeneration Treatments Trials Research Group. Baseline predictors for one-year visual outcomes with ranibizumab or bevacizumab for neovascular age-related macular degeneration. Ophthalmology. 2013;120(1):122-129.

12. Augood C, Chakravarthy U, Young I, et al. Oily fish consumption, dietary docosahexaenoic acid and eicosapentaenoic acid intakes, and associations with neovascular age-related macular degeneration. Am J Clin Nutr. 2008;88(2):398-406.

13. Delcourt C, Carrière I, Delage M, Barberger-Gateau P, Schalch W; POLA Study Group. Plasma lutein and zeaxanthin and other carotenoids as modifiable risk factors for age-related maculopathy and cataract: the POLA Study. Invest Ophthalmol Vis Sci. 2006;47(6):2329-2335.

14. Age-Related Eye Disease Study Research Group. A randomized, placebo-controlled, clinical trial of high-dose supplementation with vitamins $\mathrm{C}$ and $\mathrm{E}$, beta carotene, and zinc for age-related macular degeneration and vision loss: AREDS report no. 8. Arch Ophthalmol. 2001;119(10):1417-1436.

15. AREDS2 Research Group, Chew EY, Clemons T, SanGiovanni JP, et al. The Age-Related Eye Disease Study 2 (AREDS2): study design and baseline characteristics (AREDS2 report number 1). Ophthalmology. 2012;119(11):2282-2289.
PubMed Central and CAS, and is the official journal of The Society of Clinical Ophthalmology (SCO). The manuscript management system is completely online and includes a very quick and fair peer-review system, which is all easy to use. Visit http://www.dovepress.com/ testimonials.php to read real quotes from published authors. 